ANNALES

POLONICI MATHEMATICI

$81.1(2003)$

\title{
Jung constants of Orlicz sequence spaces
}

\author{
by TAO ZHANG (Shanghai)
}

\begin{abstract}
Estimation of the Jung constants of Orlicz sequence spaces equipped with either the Luxemburg norm or the Orlicz norm is given. The exact values of the Jung constants of a class of reflexive Orlicz sequence spaces are found by using new quantitative indices for $\mathcal{N}$-functions.
\end{abstract}

1. Preliminaries. Let $X$ be a normed linear space and $A \subset X$ be a bounded set. The diameter of $A$ is $d(A)=\sup \{\|x-y\|: x, y \in A\}$. If $z \in X$, we set $r(A, z)=\sup \{\|x-z\|: x \in A\}$. For $A, B \subset X, r(A, B)=\inf \{r(A, z)$ : $z \in B\}$ is the relative Chebyshev radius of $A$ (with respect to $B$ ), and the Chebyshev center of $A$ with respect to $B$ is defined by $z(A, B)=\{y \in B$ : $\sup \{\|x-y\|: x \in A\}=r(A, B)\}$. Clearly, $r(A, z)=r(\overline{\mathrm{co}}(A), z), r(A, B)=$ $r(\overline{\mathrm{co}}(A), B)$ and $r(A, X)=r(\overline{\mathrm{co}}(A), X)$.

Definition 1.1 (Jung [7]). The Jung constant $\mathrm{JC}(X)$ of a normed linear space $X$ is defined to be

$$
\mathrm{JC}(X)=\sup \left\{\frac{r(A, X)}{d(A)}: A \subset X \text { bounded, } d(A)>0\right\} .
$$

Clearly, always $1 / 2 \leq \operatorname{JC}(X) \leq 1$. Pichugov [12] computed $\operatorname{JC}\left(l^{p}\right)$ (see also Corollary 3.4 in Section 3). Amir [1] proved that if $X$ is a dual space, then

$$
\operatorname{JC}(X)=\sup \left\{\frac{r(A, X)}{d(A)}: A \subset X \text { finite, } d(A)>0\right\} .
$$

By using (2), Amir obtained the following.

Lemma 1.2 (see [1, Proposition 2.5(b)]). Let $\left(X_{\alpha}\right)_{\alpha \in D}$ be a net of linear subspaces of the Banach space $X$, directed by inclusion, such that $\overline{\bigcup_{\alpha \in D} X_{\alpha}}$ $=X$. If $X$ is a dual space and each $X_{\alpha}$ admits a norm-1 linear projection $P_{\alpha}$, then $\operatorname{JC}(X)=\sup _{\alpha \in D} \operatorname{JC}\left(X_{\alpha}\right)=\lim _{\alpha \in D} \operatorname{JC}\left(X_{\alpha}\right)$.

2000 Mathematics Subject Classification: Primary 46B30.

Key words and phrases: Orlicz sequence space, Jung constants, quantitative index. 
Lemma 1.3 (Pichugov [12]). Let $X_{n}$ be a real n-dimensional normed space and let $A$ be a bounded closed convex set in $X_{n}$ with $r\left(A, X_{n}\right)$ being its Chebyshev radius. Then the point $x$ is the Chebyshev center of $A$ if and only if there exists an integer $N \leq n+1$ for which

(a) there are $x_{i} \in A, i \leq N$, such that $\left\|x_{i}-x\right\|=r\left(A, X_{n}\right)$ for all $i \leq N$;

(b) there are $f_{i} \in X_{n}^{*}$, the dual space of $X_{n}, i \leq N$, such that $\left\|f_{i}\right\|=1$ and $\left\langle x_{i}-x, f_{i}\right\rangle=\left\|x_{i}-x\right\|$ for all $i \leq N$;

(c) there are $c_{i} \geq 0, i \leq N$, such that $\sum_{i=1}^{N} c_{i}=1$ and $\sum_{i=1}^{N} c_{i} f_{i}=0$.

In this case, $\sum_{i=1}^{N} \sum_{j=1}^{N} c_{i} c_{j}\left\langle x_{i}-x_{j}, f_{i}-f_{j}\right\rangle=2 r\left(A, X_{n}\right)$. If $1 \leq \lambda \leq 2$ and

$$
\Lambda=\sum_{i=1}^{N} \sum_{j=1}^{N} c_{i} c_{j}\left\{\left\langle x_{i}-x_{j}, f_{i}-f_{j}\right\rangle\right\}^{\lambda}
$$

then

$$
\frac{2^{\lambda}\left[r\left(A, X_{n}\right)\right]^{\lambda}}{\left(\frac{n}{n+1}\right)^{\lambda-1}} \leq \Lambda \leq[d(A)]^{\lambda} \sum_{i=1}^{N} \sum_{j=1}^{N} c_{i} c_{j}\left\|f_{i}-f_{j}\right\|^{\lambda} .
$$

Lemma 1.4 (Pichugov [12]). Let $X$ be a separable and dual space. If $\left\{x_{1}, x_{2}, \ldots\right\}$ is a dense set in $X$ and $X_{n}=\operatorname{span}\left\{x_{i}: 1 \leq i \leq n\right\}$, then

$$
\mathrm{JC}(X) \leq \liminf _{n \rightarrow \infty} \mathrm{JC}\left(X_{n}\right) .
$$

Recall that Bynum [2] defined the normal structure coefficient $N(X)$ of a Banach space $X$ by

$$
N(X)=\inf \left\{\frac{d(A)}{r(A, A)}: A \subset X \text { closed bounded convex, } d(A)>0\right\} .
$$

Maluta [11] denoted $[N(X)]^{-1}$ by $\widetilde{N}(X)$ and proved that $2^{-1 / 2} \leq \widetilde{N}(X)$ for every infinite-dimensional Banach space $X$. Amir [1] pointed out that for every Banach space $X$,

$$
1 / 2 \leq \mathrm{JC}(X) \leq \tilde{N}(X) \leq 1 .
$$

Next we introduce some basic facts on Orlicz spaces. Let

$$
\Phi(u)=\int_{0}^{|u|} \phi(t) d t \quad \text { and } \quad \Psi(v)=\int_{0}^{|v|} \psi(s) d s
$$

be a pair of complementary $\mathcal{N}$-functions. The Orlicz sequence space $l^{\Phi}$ is defined to be the set

$$
l^{\Phi}=\left\{x=(x(1), x(2), \ldots): \varrho_{\Phi}(\lambda x)=\sum_{i=1}^{\infty} \Phi(\lambda|x(i)|)<\infty \text { for some } \lambda>0\right\} .
$$


The Luxemburg norm and the Orlicz norm are defined respectively by

$$
\|x\|_{(\Phi)}=\inf \left\{c>0: \varrho_{\Phi}(x / c) \leq 1\right\}
$$

and

$$
\begin{aligned}
\|x\|_{\Phi} & =\sup \left\{\sum_{i=1}^{\infty}|x(i) y(i)|: \varrho_{\Psi}(y)=\sum_{i=1}^{\infty} \Psi(|y(i)|) \leq 1\right\} \\
& =\inf _{k>0} \frac{1}{k}\left[1+\varrho_{\Phi}(k x)\right] .
\end{aligned}
$$

The norms are equivalent: $\|x\|_{(\Phi)} \leq\|x\|_{\Phi} \leq 2\|x\|_{(\Phi)}$. The closed separable subspace $h^{\Phi}$ of $l^{\Phi}$ is defined to be the set

$$
h^{\Phi}=\left\{x \in l^{\Phi}: \varrho_{\Phi}(\lambda x)=\sum_{i=1}^{\infty} \Phi(\lambda|x(i)|)<\infty \text { for any } \lambda>0\right\} .
$$

An important parameter for analysis in an Orlicz space is the rate of growth of the underlying $\mathcal{N}$-function. An $\mathcal{N}$-function $\Phi(u)$ is said to satisfy the $\Delta_{2}$-condition for large $u$ (resp. for small $u$, for all $u \geq 0$ ), in symbols $\Phi \in \Delta_{2}(\infty)$ (resp. $\left.\Phi \in \Delta_{2}(0), \Phi \in \Delta_{2}\right)$, if there exist $u_{0}>0$ and $K>2$ such that $\Phi(2 u) \leq K \Phi(u)$ for $u \geq u_{0}$ (resp. for $0<u \leq u_{0}$, for $u \geq 0$ ). An $\mathcal{N}$-function $\Phi(u)$ is said to satisfy the $\nabla_{2}$-condition for large $u$, in symbols $\Phi \in \nabla_{2}(\infty)$, if there exist $u_{0}>0$ and $a>1$ such that $\Phi(u) \leq \frac{1}{2 a} \Phi(a u)$ for $u \geq u_{0}$. Similarly we define $\Phi \in \nabla_{2}(0)$ and $\Phi \in \nabla_{2}$. The basic facts on Orlicz spaces can be found in [9], [10] and [13]. For instance, $l^{\Phi}$ is separable if and only if $\Phi \in \Delta_{2}(0) ; l^{\Phi}$ is reflexive if and only if $\Phi \in \Delta_{2}(0) \cap \nabla_{2}(0)$.

New quantitative indices of $\Phi(u)$ are provided by the following six constants:

$$
\begin{array}{ll}
\alpha_{\Phi}=\liminf _{u \rightarrow \infty} \frac{\Phi^{-1}(u)}{\Phi^{-1}(2 u)}, & \beta_{\Phi}=\limsup _{u \rightarrow \infty} \frac{\Phi^{-1}(u)}{\Phi^{-1}(2 u)}, \\
\alpha_{\Phi}^{0}=\liminf _{u \rightarrow 0} \frac{\Phi^{-1}(u)}{\Phi^{-1}(2 u)}, & \beta_{\Phi}^{0}=\limsup _{u \rightarrow 0} \frac{\Phi^{-1}(u)}{\Phi^{-1}(2 u)},
\end{array}
$$

and

$$
\begin{aligned}
& \bar{\alpha}_{\Phi}=\inf \left\{\frac{\Phi^{-1}(u)}{\Phi^{-1}(2 u)}: 0<u<\infty\right\}, \\
& \bar{\beta}_{\Phi}=\sup \left\{\frac{\Phi^{-1}(u)}{\Phi^{-1}(2 u)}: 0<u<\infty\right\} .
\end{aligned}
$$

The following result will play a leading role in this paper.

Theorem 1.5. (i) $\Phi \notin \Delta_{2}(\infty) \Leftrightarrow \beta_{\Phi}=1 ; \Phi \notin \nabla_{2}(\infty) \Leftrightarrow \alpha_{\Phi}=1 / 2$;

(ii) $\Phi \notin \Delta_{2}(0) \Leftrightarrow \beta_{\Phi}^{0}=1 ; \Phi \notin \nabla_{2}(0) \Leftrightarrow \alpha_{\Phi}^{0}=1 / 2$;

(iii) $\Phi \notin \Delta_{2} \Leftrightarrow \bar{\beta}_{\Phi}=1 ; \Phi \notin \nabla_{2}(\infty) \Leftrightarrow \bar{\alpha}_{\Phi}=1 / 2$.

The proof of Theorem 1.5 can be found in [13, p. 23]. 
Other well known quantitative indices of $\Phi$ are provided by the following six constants:

$$
\begin{gathered}
A_{\Phi}=\liminf _{t \rightarrow \infty} \frac{t \phi(t)}{\Phi(t)}, \quad B_{\Phi}=\limsup _{t \rightarrow 0} \frac{t \phi(t)}{\Phi(t)} \\
A_{\Phi}^{0}=\liminf _{t \rightarrow 0} \frac{t \phi(t)}{\Phi(t)}, \quad B_{\Phi}^{0}=\limsup _{t \rightarrow 0} \frac{t \phi(t)}{\Phi(t)} \\
\bar{A}_{\Phi}=\inf \left\{\frac{t \phi(t)}{\Phi(t)}: 0<t<\infty\right\}, \quad \bar{B}_{\Phi}=\sup \left\{\frac{t \phi(t)}{\Phi(t)}: 0<t<\infty\right\} .
\end{gathered}
$$

It is also known that $\Phi \notin \Delta_{2}(\infty) \Leftrightarrow B_{\Phi}=\infty ; \Phi \notin \nabla_{2}(\infty) \Leftrightarrow A_{\Phi}=1$; $\Phi \notin \Delta_{2}(0) \Leftrightarrow A_{\Phi}^{0}=1 ; \Phi \notin \Delta_{2} \Leftarrow \bar{B}_{\Phi}=\infty$; and $\Phi \notin \nabla_{2} \Leftarrow \bar{A}_{\Phi}=1$. Furthermore, we have the following.

Proposition 1.6. Let $\Phi$ and $\Psi$ be a pair of complementary $\mathcal{N}$-functions. Then

$$
\frac{1}{A_{\Phi}}+\frac{1}{B_{\Psi}}=\frac{1}{A_{\Phi}^{0}}+\frac{1}{B_{\Psi}^{0}}=\frac{1}{\bar{A}_{\Phi}}+\frac{1}{\bar{B}_{\Psi}}=1
$$

Proposition 1.7. Let $\Phi(u)$ be an $\mathcal{N}$-function. Then

$$
\begin{aligned}
& 2^{-1 / A_{\Phi}} \leq \alpha_{\Phi} \leq \beta_{\Phi} \leq 2^{-1 / B_{\Phi}}, \\
& 2^{-1 / A_{\Phi}^{0}} \leq \alpha_{\Phi}^{0} \leq \beta_{\Phi}^{0} \leq 2^{-1 / B_{\Phi}^{0}}, \\
& 2^{-1 / \bar{A}_{\Phi}} \leq \bar{\alpha}_{\Phi} \leq \bar{\beta}_{\Phi} \leq 2^{-1 / \bar{B}_{\Phi}} .
\end{aligned}
$$

The proofs of Propositions 1.6 and 1.7 can be found in [13, p. 27].

Finally, we need some properties of Hadamard matrices, which can found in [12], [6] and [5]. The Hadamard matrix $H_{(n+1) \times(n+1)}$ of order $n+1$ is defined to be a square matrix with entries \pm 1 and with pairwise orthogonal rows. $H_{(n+1) \times(n+1)}$ is said to be in normalized form if its first column and row consist only of ones. Removing the first column of $H_{(n+1) \times(n+1)}$, we obtain the matrix $H_{n \times(n+1)}$, which is used in [12] and [6, Lemma 2].

Theorem 1.8. Let $\Phi$ and $\Psi$ be a pair of complementary $\mathcal{N}$-functions. Then

$$
2 \alpha_{\Phi}^{0} \beta_{\Psi}^{0}=1=2 \alpha_{\Psi}^{0} \beta_{\Phi}^{0}
$$

EXAMPLE 1.9. If $n+1=4$, then

$$
H_{4 \times 4}=\left[\begin{array}{rrrr}
1 & 1 & 1 & 1 \\
1 & 1 & -1 & -1 \\
1 & -1 & 1 & -1 \\
1 & -1 & -1 & 1
\end{array}\right] \quad \text { and } \quad H_{3 \times 4}=\left[\begin{array}{rrrr}
1 & 1 & -1 & -1 \\
1 & -1 & 1 & -1 \\
1 & -1 & -1 & 1
\end{array}\right] \text {. }
$$

Let $\Phi, \Psi$ be a pair of complementary $\mathcal{N}$-functions. Then $l^{(\Phi)}(3)=$ $\operatorname{span}\left\{e_{i}: 1 \leq i \leq 3\right\}$ is a subspace of $l^{(\Phi)}$, where $e_{i}=(\overbrace{0,0, \ldots, 1}^{i}, 0, \ldots)$. 
We denote by

$$
\left[x_{1}, x_{2}, x_{3}, x_{4}\right]=\Phi^{-1}(1 / 3)\left(e_{1}, e_{2}, e_{3}\right) H_{3 \times 4}
$$

the fact that

$$
\begin{aligned}
& x_{1}=\Phi^{-1}(1 / 3)\left(e_{1}+e_{2}+e_{3}\right)=\Phi^{-1}(1 / 3)(1,1,1,0,0, \ldots), \\
& x_{2}=\Phi^{-1}(1 / 3)\left(e_{1}-e_{2}-e_{3}\right)=\Phi^{-1}(1 / 3)(1,-1,-1,0,0, \ldots), \\
& x_{3}=\Phi^{-1}(1 / 3)\left(-e_{1}+e_{2}-e_{3}\right)=\Phi^{-1}(1 / 3)(-1,-1,1,0,0, \ldots), \\
& x_{4}=\Phi^{-1}(1 / 3)\left(-e_{1}-e_{2}+e_{3}\right)=\Phi^{-1}(1 / 3)(-1,-1,1,0,0, \ldots) .
\end{aligned}
$$

Then

$$
\left\|x_{i}\right\|_{(\Phi)}=\Phi^{-1}(1 / 3)\left\| \pm e_{1} \pm e_{2} \pm e_{3}\right\|_{(\Phi)}=1,
$$

where $1 \leq i \leq 4$ and for $i \neq j$,

$$
\left\|x_{i}-x_{j}\right\|_{(\Phi)}=\frac{2 \Phi^{-1}(1 / 3)}{\Phi^{-1}(1 / 2)} .
$$

Hence

$$
d\left(A_{4}\right)=\frac{2 \Phi^{-1}(1 / 3)}{\Phi^{-1}(1 / 2)} .
$$

Put

$$
y_{i}=\frac{x_{i}}{3\left[\Phi^{-1}(1 / 3)\right]^{2}}, \quad 1 \leq i \leq 4 .
$$

Then $\left\|y_{i}\right\|_{\Psi}=1, y_{i} \in l^{\Psi}(3)=\left(l^{(\Phi)}\right)^{*}$. Since $\left\| \pm e_{1} \pm e_{2} \pm e_{3}\right\|_{\Psi}=3 \Psi^{-1}(1 / 3)$, if we put $c_{i}=1 / 4$ then $\sum_{i=1}^{4} c_{i} y_{i}=0$ and $\sum_{i=1}^{4} c_{i}=1$. Therefore, by Lemma 1.3 , the set $A_{4}$ has zero as its Chebyshev center in $l^{(\Phi)}(3)=\operatorname{span}\left\{e_{i}: 1 \leq\right.$ $i \leq 3\} \subset l^{(\Phi)}$ (see also [4, Lemma 2]). It follows from (1) that

$$
\operatorname{JC}\left(l^{(\Phi)}(3)\right) \geq \frac{r\left(A_{4}, l^{(\Phi)}(3)\right)}{d\left(A_{4}\right)}=\frac{\Phi^{-1}(1 / 2)}{2 \Phi^{-1}(1 / 3)} .
$$

In general, if for some $n_{0}$,

$$
n_{0}+1 \in D=\left\{n+1 \in \mathbb{N} \text { : the Hadamard matrix } H_{(n+1) \times(n+1)} \text { exists }\right\},
$$

then put

$$
A_{n_{0}+1}=\left\{x_{i}: 1 \leq i \leq n_{0}+1\right\}, \quad l^{(\Phi)}\left(n_{0}\right)=\operatorname{span}\left\{e_{i}: 1 \leq i \leq n_{0}\right\},
$$

where

$$
\left[x_{1}, \ldots, x_{n_{0}}, x_{n_{0}+1}\right]=\Phi^{-1}\left(1 / n_{0}\right)\left[e_{1}, \ldots, e_{n_{0}}\right] H_{n_{0} \times\left(n_{0}+1\right)} .
$$

Then $\left\|x_{i}\right\|_{(\Phi)}=1, i \leq n_{0}+1$. For $i \notin j$,

$$
d\left(A_{n_{0}+1}\right)=\left\|x_{i}-x_{j}\right\|_{(\Phi)}=\frac{2 \Phi^{-1}\left(\frac{1}{n_{0}}\right)}{\Phi^{-1}\left(\frac{2}{n_{0}+1}\right)} .
$$


Finally, it follows from Lemma 1.3 that

$$
\mathrm{JC}\left(l^{(\Phi)}\left(n_{0}\right)\right) \geq \frac{r\left(A_{n_{0}+1}, l^{(\Phi)}\left(n_{0}\right)\right)}{d\left(A_{n_{0}+1}\right)}=\frac{\Phi^{-1}\left(\frac{2}{n_{0}+1}\right)}{2 \Phi^{-1}\left(\frac{1}{n_{0}}\right)} .
$$

Lemma 1.10. For an $\mathcal{N}$-function $\Phi \in \Delta_{2}(0) \cap \nabla_{2}(0)$, we have

$$
\widetilde{N}\left(l^{(\Phi)}\right)<1 \text { and } \tilde{N}\left(l^{\Phi}\right)<1 .
$$

Corollary 1.11. If an $\mathcal{N}$-function $\Phi$ is in $\Delta_{2}(0) \cap \nabla_{2}(0)$ then

$$
\max \left\{\operatorname{JC}\left(l^{(\Phi)}\right), \operatorname{JC}\left(l^{\Phi}\right)\right\}<1 .
$$

\section{Lower bounds of $\mathrm{JC}\left(l^{(\Phi)}\right)$}

Theorem 2.1. For any $\mathcal{N}$-function $\Phi$, we have

$$
\begin{aligned}
& \beta_{\Phi}^{0} \leq \operatorname{JC}\left(h^{(\Phi)}\right), \\
& \beta_{\Phi}^{0} \leq \operatorname{JC}\left(l^{(\Phi)}\right) .
\end{aligned}
$$

Proof. Since $\beta_{\Phi}^{0}=\limsup _{u \rightarrow 0} \frac{\Phi^{-1}(u)}{\Phi^{-1}(2 u)}$, there exist $1 / 2>u_{k} \searrow 0$ such that

$$
\lim _{k \rightarrow \infty} \frac{\Phi^{-1}\left(u_{k}\right)}{\Phi^{-1}\left(2 u_{k}\right)}=\beta_{\Phi}^{0}
$$

For any given $0<\varepsilon<1 / 2$, there is a $u_{0} \in\left\{u_{k}: k \geq 1\right\}$ such that

$$
u_{0}<\varepsilon / 4
$$

and

$$
\frac{\Phi^{-1}\left(u_{0}\right)}{\Phi^{-1}\left(2 u_{0}\right)}>\beta_{\Phi}^{0}-\varepsilon
$$

Put $k_{0}=\left[1 /\left(2 u_{0}\right)\right]$. Then $k_{0} \leq 1 /\left(2 u_{0}\right)<k_{0}+1$, and thus

$$
u_{0} \leq \frac{1}{2 k_{0}} \text { and } \frac{1}{k_{0}}<\frac{2 u_{0}}{1-2 u_{0}} .
$$

By (18) we have $2 u_{0}<\varepsilon / 2<\varepsilon /(1+\varepsilon)$, and so

$$
1-2 u_{0}>1-\frac{\varepsilon}{1+\varepsilon}=\frac{1}{1+\varepsilon} \text {. }
$$

We first show (16). Put

$$
C_{0}=\Phi^{-1}\left(1 / k_{0}\right)
$$

Put $X_{0}=(\overbrace{C_{0}, \ldots, C_{0}}^{k_{0}}), Y_{0}=(\overbrace{0, \ldots, 0}^{k_{0}})$, where $\operatorname{dim} X_{0}=\operatorname{dim} Y_{0}=k_{0}$. Set $x_{1}=\left(X_{0}, Y_{0}, Y_{0}, \ldots\right), x_{2}=(\overbrace{Y_{0}, X_{0}}^{2}, Y_{0}, \ldots), \ldots, x_{i}=(\overbrace{Y_{0}, \ldots, Y_{0}, X_{0}}^{i}, 0,0, \ldots)$, 
i.e.,

$$
x_{i}=C_{0} \chi\left[1+(i-1) k_{0}, i k_{0}\right] .
$$

For $n<m$, we define

$$
\chi[n, m]=\overbrace{\overbrace{0, \ldots, 0,1}^{n}, 1, \ldots, 1,0,0, \ldots) .}^{m},
$$

For any $z=(z(1), z(2), \ldots) \in h^{(\Phi)}$, we define

$$
z \chi[n, m]=\{0, \ldots, 0, z(n), z(n+1), \ldots, z(m), 0, \ldots\} .
$$

We have $A=\left\{x_{i}: i \geq 1\right\} \subset S\left(h^{(\Phi)}\right)$, since

$$
\left\|x_{i}\right\|_{(\Phi)}=\frac{C_{0}}{\Phi^{-1}\left(\frac{1}{k_{0}}\right)}=1, \quad i \geq 1 .
$$

For $i \neq j$, by (19)-(21), we have

$$
\begin{aligned}
\left\|x_{i}-x_{j}\right\|_{(\Phi)} & =\frac{\Phi^{-1}\left(\frac{1}{k_{0}}\right)}{\Phi^{-1}\left(\frac{1}{2 k_{0}}\right)}<\frac{\Phi^{-1}\left(\frac{2 u_{0}}{1-2 u_{0}}\right)}{\Phi^{-1}\left(u_{0}\right)}<\frac{\Phi^{-1}\left((1+\varepsilon) 2 u_{0}\right)}{\Phi^{-1}\left(u_{0}\right)} \\
& <\frac{(1+\varepsilon) \Phi^{-1}\left(2 u_{0}\right)}{\Phi^{-1}\left(u_{0}\right)}<\frac{1+\varepsilon}{\beta_{\Phi}^{0}-\varepsilon} .
\end{aligned}
$$

Hence

$$
d(A)<\frac{1+\varepsilon}{\beta_{\Phi}^{0}-\varepsilon} .
$$

For any $z=(z(1), z(2), \ldots) \in h^{(\Phi)}$, put

$$
P_{n} z=(z(1), \ldots, z(n), 0,0, \ldots) .
$$

Then

$$
\left\|z-P_{n} z\right\|_{(\Phi)} \rightarrow 0 \quad \text { as } n \rightarrow \infty .
$$

Since

$$
\left\|P_{m} z-P_{n} z\right\|_{(\Phi)} \leq\left\|P_{m} z-z\right\|_{(\Phi)}+\left\|z-P_{n} z\right\|_{(\Phi)}
$$

we get

$$
\lim _{n, m \rightarrow \infty}\left\|P_{m} z-P_{n} z\right\|_{(\Phi)}=0
$$

Hence

$$
\left\|z \chi\left[1+(i-1) k_{0}, i k_{0}\right]\right\|_{(\Phi)}=\left\|P_{i k_{0}} z-P_{(i-1) k_{0}} z\right\|_{(\Phi)} \rightarrow 0 \quad(i \rightarrow \infty) .
$$


Then

$$
\begin{aligned}
r(A, z) & =\sup _{i \geq 1}\left\|x_{i}-z\right\|_{(\Phi)} \geq \limsup _{i \rightarrow \infty}\left\|x_{i}-z\right\|_{(\Phi)} \\
& \geq \limsup _{i \rightarrow \infty}\left\|\left(x_{i}-z\right) \chi\left[1+(i-1) k_{0}, i k_{0}\right]\right\|_{(\Phi)} \\
& =\limsup _{i \rightarrow \infty}\left\|x_{i}-z \chi\left[1+(i-1) k_{0}, i k_{0}\right]\right\|_{(\Phi)} \\
& \geq \limsup _{i \rightarrow \infty}\left\{\left\|x_{i}\right\|_{(\Phi)}-\left\|z \chi\left[1+(i-1) k_{0}, i k_{0}\right]\right\|_{(\Phi)}\right\}=1 .
\end{aligned}
$$

We see that $r\left(A, h^{(\Phi)}\right)=\inf \left\{r(A, z): z \in h^{(\Phi)}\right\} \geq 1$. Since $z \in h^{(\Phi)}$ is arbitrary, by (23) and the definition of $\mathrm{JC}(X)$ we have

$$
\mathrm{JC}\left(h^{(\Phi)}\right) \geq \frac{r\left(A, h^{(\Phi)}\right)}{d(A)}>\frac{\beta_{\Phi}^{0}-\varepsilon}{1+\varepsilon} .
$$

We have thus proved (16) since $\varepsilon$ is arbitrary.

Next we show (17). For any $z=\{z(j)\} \in l^{(\Phi)}$, we have $\lim _{i \rightarrow \infty} \sup _{j>i} z(j)$ $=0$. Since $l^{(\Phi)} \subset c_{0}($ Chen [3, p. 169]), it follows that

$$
\begin{aligned}
r(A, z) & =\sup _{i \geq 1}\left\|x_{i}-z\right\|_{(\Phi)} \geq \limsup _{i \rightarrow \infty}\left\|x_{i}-z\right\|_{(\Phi)} \\
& \geq \limsup _{i \rightarrow \infty}\left\|\left(x_{i}-z\right) \chi\left[1+(i-1) k_{0}, i k_{0}\right]\right\|_{(\Phi)} \\
& \geq \limsup _{i \rightarrow \infty}\left\{\left\|x_{i}\right\|_{(\Phi)}-\sup _{1+(i-1) k_{0} \leq j \leq i k_{0}}|z(j)|\left\|\chi\left[1+(i-1) k_{0}, i k_{0}\right]\right\|_{(\Phi)}\right\} \\
& =1-\frac{1}{\Phi^{-1}\left(1 / k_{0}\right)} \lim _{i \rightarrow \infty} \sup _{1+(i-1) k_{0} \leq j}|z(j)|=1,
\end{aligned}
$$

proving (17).

Corollary 2.2. (i) For any $\mathcal{N}$-function $\Phi \notin \Delta_{2}(0)$, we have

$$
\mathrm{JC}\left(l^{(\Phi)}\right)=\mathrm{JC}\left(h^{(\Phi)}\right)=1 \text {. }
$$

(ii) For any $\mathcal{N}$-function $\Phi$, we have $\mathrm{JC}\left(l^{(\Phi)}\right)=\mathrm{JC}\left(h^{(\Phi)}\right)$.

Proof. (i) If $\Phi \notin \Delta_{2}(0)$, then $\beta_{\Phi}^{0}=1$. By Theorem 2.1 we have $1 \leq$ $\mathrm{JC}\left(l^{(\Phi)}\right), 1 \leq \mathrm{JC}\left(h^{(\Phi)}\right)$. For any Banach space $X$, we have $1 / 2 \leq \mathrm{JC}(X) \leq 1$. Hence (i) always holds.

(ii) For any $\mathcal{N}$-function $\Phi$, either $\Phi \notin \Delta_{2}(0)$, or $\Phi \in \Delta_{2}(0)$. If $\Phi \notin \Delta_{2}(0)$ then by (i) we have (ii); if $\Phi \in \Delta_{2}(0)$ we get $h^{(\Phi)}=l^{(\Phi)}$, hence $\operatorname{JC}\left(l^{(\Phi)}\right)=$ $\mathrm{JC}\left(h^{(\Phi)}\right)$.

TheOREM 2.3. If $\Phi \in \Delta_{2}(0)$, then

$$
\frac{1}{2 \alpha_{\Phi}^{0}} \leq \mathrm{JC}\left(l^{(\Phi)}\right) .
$$


Proof. By definition of $\alpha_{\Phi}^{0}$, for any $n \in \mathbb{N}$ and $n \geq 2$, there are $0<u_{n}<$ $1 /\left(n 2^{n}\right)$ such that

$$
\frac{\Phi^{-1}\left(u_{n}\right)}{\Phi^{-1}\left(2 u_{n}\right)}<\alpha_{\Phi}^{0}+\frac{1}{n} .
$$

Let $k_{n}=\left[1 /\left(2^{n} u_{n}\right)\right]$. Then $k_{n} \leq 1 /\left(2^{n} u_{n}\right)<k_{n}+1$. Since $2^{n} u_{n}<1 / n$, we have

$$
1-1 / n<1-2^{n} u_{n}<k_{n} 2^{n} u_{n} \leq 1 .
$$

Define $A=\left\{x_{i}: 1 \leq i \leq 2^{n}\right\}$, where

$$
\left.\left[x_{1}, \ldots, x_{2^{n}}\right]=a_{n}\left[e_{1}, \ldots, e_{k_{n}\left(2^{n}-1\right)}\right]\left[\begin{array}{c}
H_{\left(2^{n}-1\right) \times 2^{n}} \\
\vdots \\
H_{\left(2^{n}-1\right) \times 2^{n}}
\end{array}\right]\right\} k_{n}
$$

with

$$
a_{n}=\Phi^{-1}\left(\frac{1}{k_{n}\left(2^{n}-1\right)}\right) .
$$

For $n=2, k_{n}=3, k_{n}\left(2^{n}-1\right)=9$, we have

$$
\left[x_{1}, x_{2}, x_{3}, x_{4}\right]=a_{2}\left[e_{1}, \ldots, e_{9}\right]\left[\begin{array}{rrrr}
1 & 1 & -1 & -1 \\
1 & -1 & 1 & -1 \\
1 & -1 & -1 & 1 \\
1 & 1 & -1 & -1 \\
1 & -1 & 1 & -1 \\
1 & -1 & -1 & 1 \\
1 & 1 & -1 & -1 \\
1 & -1 & 1 & -1 \\
1 & -1 & -1 & 1
\end{array}\right] \text {, }
$$

i.e.,

$$
\begin{aligned}
& x_{1}=a_{2}(1,1,1 ; 1,1,1 ; 1,1,1 ; 0, \ldots), \\
& x_{2}=a_{2}(1,-1,-1 ; 1,-1,-1 ; 1,-1,-1 ; 0, \ldots), \\
& x_{3}=a_{2}(-1,1,-1 ;-1,1,-1 ;-1,1,-1 ; 0, \ldots), \\
& x_{4}=a_{2}(-1,-1,1 ;-1,-1,1 ;-1,-1,1 ; 0, \ldots) .
\end{aligned}
$$

Hence $\left\|x_{i}\right\|_{(\Phi)}=1,1 \leq i \leq 2^{n}$. Let $\Psi$ be the complementary $\mathcal{N}$-function to $\Phi$ and

$$
y_{i}=\frac{x_{i}}{a_{n}^{2} k_{n}\left(2^{n}-1\right)}, \quad c_{i}=\frac{1}{2^{n}}, \quad 1 \leq i \leq 2^{n} .
$$

Then $\left\|y_{i}\right\|_{\Psi}=1, \sum_{i=1}^{2^{n}} c_{i} y_{i}=0,\left\langle x_{i}, y_{j}\right\rangle=0(i \neq j)$ and $\left\langle x_{i}, y_{i}\right\rangle=1=$ $\left\|x_{i}\right\|_{(\Phi)}$. Let

$$
l^{(\Phi)}\left(k_{n}\left(2^{n}-1\right)\right)=\operatorname{span}\left\{e_{i}: 1 \leq i \leq k_{n}\left(2^{n}-1\right)\right\} .
$$


By Lemma 1.3, 0 is the Chebyshev center of $A$ and

$$
r\left(A, l^{(\Phi)}\left(k_{n}\left(2^{n}-1\right)\right)\right)=1 .
$$

We will prove that

$$
\left\|x_{i}-x_{j}\right\|_{(\Phi)}=\frac{2 a_{n}}{\Phi^{-1}\left(\frac{2}{k_{n} 2^{n}}\right)}, \quad i \neq j .
$$

By (26) we have

$$
k_{n}\left(2^{n}-1\right) u_{n}>1-\frac{1}{n}-k_{n} u_{n} \geq 1-\frac{1}{n}-\frac{1}{2^{n}}
$$

and

$$
2 a_{n}=2 \Phi^{-1}\left(\frac{u_{n}}{k_{n}\left(2^{n}-1\right) u_{n}}\right)<2 \Phi^{-1}\left(\frac{u_{n}}{1-\frac{1}{n}-\frac{1}{2^{n}}}\right)<\frac{2 \Phi^{-1}\left(u_{n}\right)}{1-\frac{1}{n}-\frac{1}{2^{n}}} .
$$

On the other hand, by (26),

$$
\Phi^{-1}\left(\frac{2}{k_{n} 2^{n}}\right)=\Phi^{-1}\left(\frac{2 u_{n}}{k_{n} 2^{n} u_{n}}\right) \geq \Phi^{-1}\left(2 u_{n}\right) .
$$

By (28), (30), (31) and (25) we have

$$
d(A)<\frac{2\left(\alpha_{\Phi}^{0}+\frac{1}{n}\right)}{1-\frac{1}{n}-\frac{1}{2^{n}}} .
$$

By (27), (32) and the definition of $\operatorname{JC}(X)$ we get

$$
\mathrm{JC}\left(l^{(\Phi)}\left(k_{n}\left(2^{n}-1\right)\right)\right) \geq \frac{r\left(A, l^{(\Phi)}\left(k_{n}\left(2^{n}-1\right)\right)\right)}{d(A)}>\frac{1-\frac{1}{n}-\frac{1}{2^{n}}}{2\left(\alpha_{\Phi}^{0}+\frac{1}{n}\right)} .
$$

Since $k_{m}\left(2^{m}-1\right) \geq k_{n}\left(2^{n}-1\right)$ we have $l^{(\Phi)}\left(k_{n}\left(2^{n}-1\right)\right) \subset l^{(\Phi)}\left(k_{m}\left(2^{m}-1\right)\right)$. As $\Phi \in \Delta_{2}(0)$, it follows that

$$
\overline{\bigcup_{n \geq 2} l^{(\Phi)}\left(k_{n}\left(2^{n}-1\right)\right)}=l^{(\Phi)} .
$$

Define $P_{k_{n}\left(2^{n}-1\right)}: l^{(\Phi)} \rightarrow l^{(\Phi)}\left(k_{n}\left(2^{n}-1\right)\right)$ for $x=(x(1), x(2), \ldots) \in l^{(\Phi)}$ by

$$
P_{k_{n}\left(2^{n}-1\right)} x=\left(x(1), \ldots, x\left(k_{n}\left(2^{n}-1\right)\right), 0,0, \ldots\right) .
$$

Then $\left\|P_{k_{n}\left(2^{n}-1\right)}\right\|=1$. Moreover, $l^{(\Phi)}=\left(h^{\Psi}\right)^{*}$. Hence, by Theorem 1.3 and (33) we get (24):

$$
\begin{aligned}
\operatorname{JC}\left(l^{(\Phi)}\right) & =\sup _{n \geq 2} \operatorname{JC}\left(l^{(\Phi)}\left(k_{n}\left(2^{n}-1\right)\right)\right) \\
& \geq \sup _{n \geq 2} \frac{1-\frac{1}{n}-\frac{1}{2^{n}}}{2\left(\alpha_{\Phi}^{0}+\frac{1}{n}\right)}=\frac{1}{2 \alpha_{\Phi}^{0}} .
\end{aligned}
$$


Corollary 2.4. (i) $l^{(\Phi)}$ is not reflexive if and only if $\mathrm{JC}\left(l^{(\Phi)}\right)=1$.

(ii) If $\Phi \in \Delta_{2}(0) \cap \nabla_{2}(0)$, then

$$
\frac{1}{\sqrt{2}} \leq \max \left(\beta_{\Phi}^{0}, \frac{1}{2 \alpha_{\Phi}^{0}}\right) \leq \operatorname{JC}\left(l^{(\Phi)}\right) .
$$

Proof. (i) If $l^{(\Phi)}$ is not reflexive, then either $\Phi \notin \Delta_{2}(0)$ or $\Phi \in \Delta_{2}(0) \backslash \nabla_{2}(0)$. For $\Phi \notin \Delta_{2}(0)$, the conclusion follows from Corollary 2.2. For $\Phi \in \Delta_{2}(0) \backslash \nabla_{2}(0)$, by Theorem $1.5\left(\right.$ ii) we get $\alpha_{\Phi}^{0}=1 / 2$. By $(24), 1=1 /\left(2 \alpha_{\Phi}^{0}\right) \leq \operatorname{JC}\left(l^{(\Phi)}\right) \leq 1$. i.e., $\operatorname{JC}\left(l^{(\Phi)}\right)=1$. If $l^{(\Phi)}$ is reflexive, by Corollary 1.10 we have $\operatorname{JC}\left(l^{(\Phi)}\right)<1$.

(ii) If $\Phi \in \Delta_{2}(0) \cap \nabla_{2}(0)$, it is sufficient to show that $1 / \sqrt{2} \leq$ $\max \left(\beta_{\Phi}^{0}, 1 /\left(2 \alpha_{\Phi}^{0}\right)\right)$.

Indeed, otherwise $1 / \sqrt{2}>\beta_{\Phi}^{0}$ and $1 / \sqrt{2}>1 /\left(2 \alpha_{\Phi}^{0}\right)$. Hence $\alpha_{\Phi}^{0}>1 / \sqrt{2}$ $>\beta_{\Phi}^{0}$, a contradiction, since $\alpha_{\Phi}^{0} \leq \beta_{\Phi}^{0}$ always holds.

Now let us turn to Orlicz sequence spaces equipped with the Orlicz norm. If $\Psi$ is the complementary $\mathcal{N}$-function to $\Phi$, then

$$
\alpha_{\Psi}^{0}=\liminf _{v \rightarrow 0} \frac{\Psi^{-1}(v)}{\Psi^{-1}(2 v)} .
$$

Theorem 2.5. For any $\mathcal{N}$-function $\Phi$, we have

$$
\begin{aligned}
\frac{1}{2 \alpha_{\Psi}^{0}} & \leq \mathrm{JC}\left(h^{\Phi}\right), \\
\frac{1}{2 \alpha_{\Psi}^{0}} & \leq \mathrm{JC}\left(l^{\Phi}\right) .
\end{aligned}
$$

Proof. By (35) there exist $1 / 4>v_{k} \searrow 0$ such that

$$
\lim _{k \rightarrow \infty} \frac{\Psi^{-1}\left(v_{k}\right)}{\Psi^{-1}\left(2 v_{k}\right)}=\alpha_{\Psi}^{0}
$$

For any $0<\varepsilon<1$, exist some $v_{0} \in\left\{v_{k}: k \geq 1\right\}$ such that $v_{0}<\varepsilon / 4$ and

$$
\frac{\Psi^{-1}\left(v_{0}\right)}{\Psi^{-1}\left(2 v_{0}\right)}<\alpha_{\Psi}^{0}+\varepsilon
$$

Put $k_{0}=\left[1 /\left(2 v_{0}\right)\right]$. Then $k_{0} \leq 1 /\left(2 v_{0}\right)<k_{0}+1$, hence

$$
\frac{1}{k_{0}+1}<2 v_{0} \leq \frac{1}{k_{0}}
$$

and

$$
\frac{1}{k_{0}}<\frac{2 v_{0}}{1-2 v_{0}}<4 v_{0}<\varepsilon .
$$

Put

$$
C_{0}=\frac{1}{k_{0} \Psi^{-1}\left(1 / k_{0}\right)} .
$$


We first show (36). Define $A=\left\{x_{i}: i \geq 1\right\}$, where

$$
\begin{aligned}
& x_{1}=C_{0}(\overbrace{1, \ldots, 1}^{k_{0}}, 0,0, \ldots), \\
& x_{2}=C_{0}(\overbrace{0, \ldots, 0}^{k_{0}}, \overbrace{1, \ldots, 1}^{k_{0}}, 0,0, \ldots), \ldots,
\end{aligned}
$$

i.e., $x_{i}=C_{0} \chi\left[1+(i-1) k_{0}, i k_{0}\right]$. By (41) we have $\left\|x_{i}\right\|_{\Phi}=1(i \geq 1)$. For $0<u_{1} \leq u_{2}$

$$
\frac{\Psi^{-1}\left(u_{2}\right)}{u_{2}} \leq \frac{\Psi^{-1}\left(u_{1}\right)}{u_{1}}
$$

By $(38)-(40)$, for $i \neq j$ we have

$$
\begin{aligned}
\left\|x_{i}-x_{j}\right\|_{\Phi} & =C_{0} 2 k_{0} \Psi^{-1}\left(\frac{1}{2 k_{0}}\right) \leq C_{0} \frac{\Psi^{-1}\left(v_{0}\right)}{v_{0}} \\
& <2\left(\alpha_{\Psi}^{0}+\varepsilon\right) C_{0} \frac{\Psi^{-1}\left(2 v_{0}\right)}{2 v_{0}} \\
& <2\left(\alpha_{\Psi}^{0}+\varepsilon\right) C_{0}\left(k_{0}+1\right) \Psi^{-1}\left(\frac{1}{k_{0}+1}\right) \\
& <2\left(\alpha_{\Psi}^{0}+\varepsilon\right) C_{0}\left(k_{0}+1\right) \Psi^{-1}\left(\frac{1}{k_{0}}\right) \\
& =2\left(\alpha_{\Psi}^{0}+\varepsilon\right)\left(1+\frac{1}{k_{0}}\right)<2(1+\varepsilon)\left(\alpha_{\Psi}^{0}+\varepsilon\right),
\end{aligned}
$$

i.e.,

$$
d(A)<2(1+\varepsilon)\left(\alpha_{\Psi}^{0}+\varepsilon\right) .
$$

For any $z=(z(1), z(2), \ldots) \in h^{\Phi}$, put

$$
P_{n} z=(z(1), \ldots, z(n), 0,0, \ldots) .
$$

Then

$$
\left\|z \chi\left[1+(i-1) k_{0}, i k_{0}\right]\right\|_{\Phi}=\left\|P_{i k_{0}} z-P_{(i-1) k_{0}} z\right\|_{\Phi} \rightarrow 0, \quad i \rightarrow \infty .
$$

Hence

$$
\begin{aligned}
r(A, z) & =\sup _{i \geq 1}\left\|x_{i}-z\right\|_{\Phi} \geq \limsup _{i \rightarrow \infty}\left\|x_{i}-z\right\|_{\Phi} \\
& \geq \limsup _{i \rightarrow \infty}\left\|\left(x_{i}-z\right) \chi\left[1+(i-1) k_{0}, i k_{0}\right]\right\|_{\Phi} \\
& =\limsup _{i \rightarrow \infty}\left\|x_{i}-z \chi\left[1+(i-1) k_{0}, i k_{0}\right]\right\|_{\Phi} \\
& \geq \limsup _{i \rightarrow \infty}\left\{\left\|x_{i}\right\|_{\Phi}-\left\|z \chi\left[1+(i-1) k_{0}, i k_{0}\right]\right\|_{\Phi}\right\}=1 .
\end{aligned}
$$


Since $z \in h^{\Phi}$ is arbitrary, we have $r\left(A, h^{\Phi}\right)=\inf \left\{r(A, z): z \in h^{\Phi}\right\} \geq 1$. By (42),

$$
\mathrm{JC}\left(h^{\Phi}\right) \geq \frac{r\left(A, h^{\Phi}\right)}{d(A)}>\frac{1}{2\left(\alpha_{\Psi}^{0}+\varepsilon\right)(1+\varepsilon)} .
$$

As $\varepsilon$ is arbitrary we get (36).

Next we show (37). If $z=(z(1), z(2), \ldots) \in l^{\Phi}$, then

$$
\begin{aligned}
\| z \chi[1 & \left.+(i-1) k_{0}, i k_{0}\right] \|_{\Phi} \\
& \leq \sup \left\{|z(j)|: 1+(i-1) k_{0} \leq j \leq i k_{0}\right\}\left\|\chi\left[1+(i-1) k_{0}, i k_{0}\right]\right\|_{\Phi} \\
& \leq k_{0} \Psi^{-1}\left(\frac{1}{k_{0}}\right) \sup \left\{|z(j)|: j \geq 1+(i-1) k_{0}\right\} \rightarrow 0 \quad(i \rightarrow \infty) .
\end{aligned}
$$

Hence $r\left(A, l^{\Phi}\right) \geq 1$ and so

$$
\mathrm{JC}\left(l^{\Phi}\right) \geq \frac{1}{2(1+\varepsilon)\left(\alpha_{\Psi}^{0}+\varepsilon\right)} .
$$

Since $\varepsilon$ is arbitrary we get (37).

Corollary 2.6. (i) If $\Phi \notin \Delta_{2}(0)$, then $\operatorname{JC}\left(l^{\Phi}\right)=\mathrm{JC}\left(h^{\Phi}\right)=1$.

(ii) For any $\mathcal{N}$-function $\Phi$ we have $\operatorname{JC}\left(l^{\Phi}\right)=\mathrm{JC}\left(h^{\Phi}\right)$.

Proof. (i) If $\Phi \notin \Delta_{2}(0)$, then $\Psi \notin \nabla_{2}(0)$. Hence $\alpha_{\Psi}^{0}=1 / 2$, which implies (i) by Theorem 2.5 .

(ii) For $\Phi \notin \Delta_{2}(0)$ see (i). For $\Phi \in \Delta_{2}(0)$ we have $h^{\Phi}=l^{\Phi}$. The result follows from the proof of Theorem 2.5.

Theorem 2.7. If $\Phi \in \Delta_{2}(0)$, then

$$
\beta_{\Psi}^{0} \leq \mathrm{JC}\left(l^{\Phi}\right),
$$

where $\Psi$ is the complementary $\mathcal{N}$-function to $\Phi$ and

$$
\beta_{\Psi}^{0}=\limsup _{v \rightarrow 0} \frac{\Psi^{-1}(v)}{\Psi^{-1}(2 v)} .
$$

Proof. By definition of $\beta_{\Psi}^{0}$, for any $n \in \mathbb{N}$ and $n \geq 2$, there exist $0<$ $v_{n}<1 /\left(n 2^{n}\right)$ such that

$$
\frac{\Psi^{-1}\left(v_{n}\right)}{\Psi^{-1}\left(2 v_{n}\right)}>\beta_{\Psi}^{0}-\frac{1}{n} .
$$

Let $k_{n}=\left[1 /\left(2^{n} v_{n}\right)\right]$. Then $k_{n} \leq 1 /\left(2^{n} v_{n}\right)<k_{n}+1$, hence

$$
1-1 / n<1-2^{n} v_{n}<k_{n} 2^{n} v_{n} \leq 1 .
$$


Define $B=\left\{x_{i}: 1 \leq i \leq 2^{n}\right\}$, where

$$
\left.\left[x_{1}, \ldots, x_{2^{n}}\right]=b_{n}\left[e_{1}, \ldots, e_{k_{n}\left(2^{n}-1\right)}\right]\left[\begin{array}{c}
H_{\left(2^{n}-1\right) \times 2^{n}} \\
\vdots \\
H_{\left(2^{n}-1\right) \times 2^{n}}
\end{array}\right]\right\} k_{n}
$$

and

$$
b_{n}=\frac{1}{k_{n}\left(2^{n}-1\right) \Psi^{-1}\left(\frac{1}{k_{n}\left(2^{n}-1\right)}\right)} .
$$

Hence $\left\|x_{i}\right\|_{\Phi}=1,1 \leq i \leq 2^{n}$. We have

$$
r\left(B, l^{\Phi}\left(k_{n}\left(2^{n}-1\right)\right)\right)=1 .
$$

We will prove that

$$
\left\|x_{i}-x_{j}\right\|_{\Phi}=2 b_{n}\left[\frac{k_{n} 2^{n}}{2} \Psi^{-1}\left(\frac{2}{k_{n} 2^{n}}\right)\right] .
$$

By (45) we have

$$
\Psi^{-1}\left(\frac{2}{k_{n} 2^{n}}\right)=\Psi^{-1}\left(\frac{2 v_{n}}{k_{n} 2^{n} v_{n}}\right)<\Psi^{-1}\left(\frac{2 v_{n}}{1-1 / n}\right)<\frac{1}{1-1 / n} \Psi^{-1}\left(2 v_{n}\right) .
$$

By (45) we also have

$$
k_{n}\left(2^{n}-1\right) v_{n}=k_{n} 2^{n} v_{n}-k_{n} v_{n} \leq 1-k_{n} v_{n}<1-\frac{1}{2^{n}}\left(1-\frac{1}{n}\right)<1 .
$$

Hence

$$
\Psi^{-1}\left(\frac{1}{k_{n}\left(2^{n}-1\right)}\right)=\Psi^{-1}\left(\frac{v_{n}}{k_{n}\left(2^{n}-1\right) v_{n}}\right)>\Psi^{-1}\left(v_{n}\right)
$$

and

$$
\begin{aligned}
d(B) & <\frac{k_{n} 2^{n} \Psi^{-1}\left(2 v_{n}\right)}{k_{n}\left(2^{n}-1\right)(1-1 / n) \Psi^{-1}\left(v_{n}\right)} \\
& <\frac{1}{\left(1-1 / 2^{n}\right)(1-1 / n)\left(\beta_{\Psi}^{0}-1 / n\right)} .
\end{aligned}
$$

Hence

$$
\begin{aligned}
J\left(l^{\Phi}\left(k_{n}\left(2^{n}-1\right)\right)\right) & \geq \frac{r\left(B, l^{\Phi}\left(k_{n}\left(2^{n}-1\right)\right)\right)}{d(B)} \\
& \geq\left(1-\frac{1}{2^{n}}\right)\left(1-\frac{1}{n}\right)\left(\beta_{\Psi}^{0}-\frac{1}{n}\right) .
\end{aligned}
$$

Since $\Phi \in \Delta_{2}(0)$ we get

$$
\overline{\bigcup_{n \geq 2} l^{\Phi}\left(k_{n}\left(2^{n}-1\right)\right)}=l^{\Phi} .
$$


By Lemma 1.2 and (48) we get (43):

$$
\begin{aligned}
\mathrm{JC}\left(l^{\Phi}\right) & =\sup _{n \geq 2} \mathrm{JC}\left(l^{\Phi}\left(k_{n}\left(2^{n}-1\right)\right)\right) \\
& \geq \sup _{n \geq 2}\left(1-\frac{1}{2^{n}}\right)\left(1-\frac{1}{n}\right)\left(\beta_{\Psi}^{0}-\frac{1}{n}\right)=\beta_{\Psi}^{0} .
\end{aligned}
$$

Corollary 2.8. (i) $l^{\Phi}$ is not reflexive if and only if $\mathrm{JC}\left(l^{\Phi}\right)=1$.

(ii) If $\Phi \in \Delta_{2}(0) \cap \nabla_{2}(0)$, then

$$
\frac{1}{\sqrt{2}} \leq \max \left(\frac{1}{2 \alpha_{\Psi}^{0}}, \beta_{\Psi}^{0}\right) \leq \mathrm{JC}\left(l^{\Phi}\right) .
$$

Proof. (i) If $l^{\Phi}$ is not reflexive, then either $\Phi \notin \Delta_{2}(0)$ or $\Phi \in \Delta_{2}(0) \backslash \nabla_{2}(0)$. If $\Phi \notin \Delta_{2}(0)$, the argument is similar to that of Corollary 2.6(i). If $\Phi \in$ $\Delta_{2}(0) \backslash \nabla_{2}(0)$, then $\Psi \notin \Delta_{2}(0)$, hence $\beta_{\Psi}^{0}=1$. By (43) we get $\operatorname{JC}\left(l^{\Phi}\right)=1$.

(ii) If $l^{\Phi}$ is reflexive, (49) follows from the proof of Theorems 2.5 and 2.7 and Corollary 2.4.

TheOrem 2.9. Let $\Phi, \Psi$ be a pair of complementary $\mathcal{N}$-functions. If $\Phi \in$ $\Delta_{2}(0) \cap \nabla_{2}(0)$, then

$$
\max \left(\beta_{\Phi}^{0}, \frac{1}{2 \alpha_{\Phi}^{0}}\right) \leq \min \left\{\operatorname{JC}\left(l^{(\Phi)}\right), \operatorname{JC}\left(l^{\Psi}\right), \operatorname{JC}\left(l^{\Phi}\right), \operatorname{JC}\left(l^{(\Psi)}\right)\right\} .
$$

Proof. By Corollaries 2.4(ii) and 2.8(ii), if $\Phi \in \Delta_{2}(0) \cap \nabla_{2}(0)$, then

$$
\begin{aligned}
& \max \left(\beta_{\Phi}^{0}, \frac{1}{2 \alpha_{\Phi}^{0}}\right) \leq \min \left\{\operatorname{JC}\left(l^{(\Phi)}\right), \operatorname{JC}\left(l^{\Psi}\right)\right\}, \\
& \max \left(\frac{1}{2 \alpha_{\Psi}^{0}}, \beta_{\Psi}^{0}\right) \leq \min \left\{\operatorname{JC}\left(l^{\Phi}\right), \operatorname{JC}\left(l^{(\Psi)}\right)\right\} .
\end{aligned}
$$

By Theorem 1.8, $\beta_{\Phi}^{0}=1 /\left(2 \alpha_{\Psi}^{0}\right), 1 /\left(2 \alpha_{\Phi}^{0}\right)=\beta_{\Psi}^{0}$. Hence we get $(50)$.

Example 2.10. Let $1<p<\infty$ and $1 / p+1 / q=1$. Then

$$
\max \left(2^{-1 / p}, 2^{1 / p-1}\right) \leq\left\{\mathrm{JC}\left(l^{p}\right), \operatorname{JC}\left(l^{q}\right)\right\} .
$$

In fact, let $\Phi_{p}(u)=|u|^{p}$. Then $l^{\left(\Phi_{p}\right)}=l^{p}, \Phi_{p} \in \Delta_{2}(0) \cap \nabla_{2}(0)$. For $u>0$,

$$
\frac{\Phi_{p}^{-1}(u)}{\Phi_{p}^{-1}(2 u)}=\frac{u^{1 / p}}{(2 u)^{1 / p}}=2^{-1 / p} .
$$

Hence $\alpha_{\Phi_{p}}^{0}=\beta_{\Phi_{p}}^{0}=2^{-1 / p}$ and we get (53) by (51).

EXAMPLE 2.11. Let $\Phi_{r}(u)=e^{|u|^{r}}-1,1<r<\infty$ and let $\Psi_{r}$ be the complementary $\mathcal{N}$-function to $\Phi_{r}$. Then

$$
\max \left(2^{-1 / r}, 2^{1 / r-1}\right) \leq \min \left\{\operatorname{JC}\left(l^{\left(\Phi_{r}\right)}\right), \operatorname{JC}\left(l^{\Phi_{r}}\right), \operatorname{JC}\left(l^{\Psi_{r}}\right), \operatorname{JC}\left(l^{\left(\Psi_{r}\right)}\right)\right\} .
$$


In fact, $\Phi_{r}^{-1}(u)=[\ln (1+u)]^{1 / r}$. Hence

$$
\alpha_{\Phi_{r}}^{0}=\beta_{\Phi_{r}}^{0}=\gamma_{\Phi_{r}}^{0}=\lim _{u \rightarrow 0} \frac{\Phi_{r}^{-1}(u)}{\Phi_{r}^{-1}(2 u)}=2^{-1 / r} .
$$

Hence $\Phi_{r} \in \Delta_{2}(0) \cap \nabla_{2}(0)$ and we get (54) by (50).

ExAmPle 2.12. Let $\Phi(u)=e^{|u|}-|u|-1, \Psi(v)=(1+|v|) \ln (1+|v|)-|v|$. Since

$$
C_{\Phi}^{0}=\lim _{t \rightarrow 0} \frac{t \phi(t)}{\Phi(t)}=\lim _{t \rightarrow 0} \frac{t\left(e^{t}-1\right)}{e^{t}-t-1}=2
$$

we get $\Phi, \Psi \in \Delta_{2}(0) \cap \nabla_{2}(0)$ and $\alpha_{\Phi}^{0}=\beta_{\Phi}^{0}=2^{-1 / 2}$. By (50) we have

$$
\frac{1}{\sqrt{2}} \leq \min \left\{\mathrm{JC}\left(l^{(\Phi)}\right), \mathrm{JC}\left(l^{\Phi}\right), \mathrm{JC}\left(l^{(\Psi)}\right), \mathrm{JC}\left(l^{\Psi}\right)\right\}
$$

ExAMPLE 2.13. Let

$$
\Phi(u)=(1+|u|)^{\sqrt{\ln (1+|u|)}}-1 .
$$

Then

$$
C_{\Phi}^{0}=\lim _{t \rightarrow 0} \frac{t \phi(t)}{\Phi(t)}=\frac{3}{2}
$$

Hence $\Phi \in \Delta_{2}(0) \cap \nabla_{2}(0), \alpha_{\Phi}^{0}=\beta_{\Phi}^{0}=\gamma_{\Phi}^{0}=2^{-1 / C_{\Phi}^{0}}=2^{-2 / 3}$. By $(50)$,

$$
\frac{1}{\sqrt[3]{2}}=\max \left(\gamma_{\Phi}^{0}, \frac{1}{2 \gamma_{\Phi}^{0}}\right) \leq \min \left\{\mathrm{JC}\left(l^{(\Phi)}\right), \operatorname{JC}\left(l^{\Phi}\right), \operatorname{JC}\left(l^{(\Psi)}\right), \operatorname{JC}\left(l^{\Psi}\right)\right\} .
$$

3. $\mathrm{JC}\left(l^{\left(\Phi_{s}\right)}\right)$ and $\mathrm{JC}\left(l^{\Phi_{s}}\right)$

Definition 3.1. Let $\Phi$ be an $\mathcal{N}$-function. Let $\Phi_{0}(u)=u^{2}, 0<s \leq 1$, and let $\Phi_{s}$ be the inverse of

$$
\Phi_{s}^{-1}(u)=\left[\Phi^{-1}(u)\right]^{1-s}\left[\Phi_{0}^{-1}(u)\right]^{s} \quad(u \geq 0) .
$$

LEMMA 3.2. Let $\Phi$ be an $\mathcal{N}$-function.

(i) For any $0<s \leq 1, \Phi_{s} \in \Delta_{2}(0) \cap \nabla_{2}(0)$.

(ii) Let $N\left(l^{\left(\Phi_{s}\right)}\right)$ and $N\left(l^{\Phi_{s}}\right)$ be the normal structure coefficients of $l^{\left(\Phi_{s}\right)}$ and $l^{\Phi_{s}}$. Then

$$
\begin{aligned}
& 2^{s / 2} \leq N\left(l^{\left(\Phi_{s}\right)}\right), \\
& 2^{s / 2} \leq N\left(l^{\Phi_{s}}\right) .
\end{aligned}
$$

TheOREM 3.3. Let $\Phi$ be an $\mathcal{N}$-function. Let $\Psi_{s}^{+}$be the complementary $\mathcal{N}$-function to $\Phi_{s}$. Then

$$
\begin{gathered}
\max \left\{\mathrm{JC}\left(l^{\left(\Phi_{s}\right)}\right), \mathrm{JC}\left(l^{\Phi_{s}}\right)\right\} \leq 2^{-s / 2}, \\
\max \left\{\mathrm{JC}\left(l^{\left(\Psi_{s}^{+}\right)}\right), \mathrm{JC}\left(l^{\Psi_{s}^{+}}\right)\right\} \leq 2^{-s / 2} .
\end{gathered}
$$


Proof. We get (58) from (56), $(57)$ and $\operatorname{JC}(X) \leq \widetilde{N}(X)=1 / N(X)$. We now prove (59). We first show

$$
\mathrm{JC}\left(l^{\Psi_{s}^{+}}\right) \leq 2^{-s / 2} .
$$

By Lemma 3.2, $l^{\Psi_{s}^{+}}$is reflexive, and of course it is a separable dual space. Let $\left\{z_{i}: i \geq 1\right\}$ be a dense set in $l^{\Psi_{s}^{+}}$, and $X_{n}=\operatorname{span}\left\{z_{i}: 1 \leq i \leq n\right\}$. For any given bounded closed convex set $A \subset X_{n}$ with Chebyshev radius $r\left(A, X_{n}\right)$, there always exists a Chebyshev center $x$ of $A$. In view of Lemma 1.3, there exist an integer $N \leq n,\left\{x_{i}: i \leq N\right\} \subset l^{\Psi_{s}^{+}},\left\{y_{i}: i \leq N\right\} \subset S\left(\left(l^{\Psi_{s}^{+}}\right)^{*}\right)=$ $S\left(l^{\left(\Phi_{s}\right)}\right)$ and $\left\{c_{i} \geq 0: i \leq N\right\}$ and $\sum_{i=1}^{N} c_{i}=1$, which satisfy conditions (a)-(c) of Lemma 1.3. Putting $\lambda=2 /(2-s)$ in (3), we have

$$
\begin{aligned}
\frac{2^{\frac{2}{2-s}}\left[r\left(A, X_{n}\right)\right]^{\frac{2}{2-s}}}{\left(\frac{n}{n+1}\right)^{\frac{2}{2-s}-1}} & \leq[d(A)]^{\frac{2}{2-s}} \sum_{i=1}^{N} \sum_{j=1}^{N} c_{i} c_{j}\left\|y_{i}-y_{j}\right\|_{\left(\Phi_{s}\right)}^{\frac{2}{2-s}} \\
& \leq[d(A)]^{\frac{2}{2-s}} 2 \sum_{i=1}^{N} c_{i}\left\|y_{i}\right\|_{\left(\Phi_{s}\right)}^{\frac{2}{2-s}}=2[d(A)]^{\frac{2}{2-s}} .
\end{aligned}
$$

Hence

$$
\frac{r\left(A, X_{n}\right)}{d(A)} \leq 2^{-s / 2}\left(\frac{n}{n+1}\right)^{s / 2} .
$$

Since $A$ is arbitrary, we obtain

$$
\mathrm{JC}\left(X_{n}\right) \leq 2^{-s / 2}\left(\frac{n}{n+1}\right)^{s / 2} .
$$

Hence, by using Lemmas 1.4 and 3.2(i) we can prove (60):

$$
\mathrm{JC}\left(l^{\Psi_{s}^{+}}\right) \leq \liminf _{n \rightarrow \infty} 2^{-s / 2}\left(\frac{n}{n+1}\right)^{s / 2}=2^{-s / 2} .
$$

Corollary 3.4 (Ivanov and Pichugov [6]). Let $1<p<\infty$ and $1 / p+$ $1 / q=1$. Then

$$
\mathrm{JC}\left(l^{p}\right)=\mathrm{JC}\left(l^{q}\right)=\max \left(2^{1 / p-1}, 2^{-1 / p}\right) .
$$

Proof. In fact, putting $\Phi(u)=|u|^{p}$, we have $l^{(\Phi)}=l^{p},\|\cdot\|_{(\Phi)}=\|\cdot\|_{p}$, $l^{\Psi}=l^{q}$ and $\|\cdot\|_{\Psi}=\|\cdot\|_{q}$, where

$$
\Psi(v)=\frac{(q-1)^{q-1}}{q^{q}}|v|^{q}
$$

is the complementary $\mathcal{N}$-function to $\Phi(u)$. By Example 2.10, we need only prove

$$
\max \left\{\mathrm{JC}\left(l^{p}\right), \operatorname{JC}\left(l^{q}\right)\right\} \leq \max \left(2^{1 / p-1}, 2^{-1 / p}\right) .
$$


If $1<p \leq 2$, we choose $1<a<p \leq 2$. Put $\Phi(u)=|u|^{a}, \Phi_{0}(u)=u^{2}$ and $s=2(p-a) /(p(2-a))$. We have $0<s \leq 1$ and for $u \geq 0$,

$$
\Phi_{s}^{-1}(u)=u^{(1-s) / a+s / 2}=u^{1 / p},
$$

i.e., $\Phi_{s}(u)=|u|^{p}$. Since $l^{\left(\Phi_{s}\right)}=l^{p}, l^{\Psi_{s}^{+}}=l^{q}$ and $\lim _{a \searrow 1}(-s / 2)=1 / p-1$ we get

$$
\max \left\{\operatorname{JC}\left(l^{p}\right), \operatorname{JC}\left(l^{q}\right)\right\} \leq 2^{1 / p-1}, \quad 1<p \leq 2 .
$$

If $2 \leq p<\infty$, we choose $2 \leq p<b<\infty$. Let $\Phi(u)=|u|^{b}$ and $s=$ $2(b-p) /(p(b-2))$. Again we have $0<s \leq 1$ and $\Phi_{s}(u)=|u|^{p}$. Noting that $\lim _{b / \infty}(-s / 2)=-1 / p$, we get

$$
\max \left\{\mathrm{JC}\left(l^{p}\right), \mathrm{JC}\left(l^{q}\right)\right\} \leq 2^{-1 / p}, \quad 2 \leq p<\infty .
$$

Finally, (62) follows from (63) and (64).

Corollary 3.5. Let $\Phi_{s}, \Psi_{s}^{+}$be as in Theorem 3.3 with $0<s \leq 1$. Then

(65) $\max \left(\beta_{\Phi_{s}}^{0}, \frac{1}{2 \alpha_{\Phi_{s}}^{0}}\right) \leq\left\{\mathrm{JC}\left(l^{\left(\Phi_{s}\right)}\right), \operatorname{JC}\left(l^{\Phi_{s}}\right), \operatorname{JC}\left(l^{\Psi_{s}^{+}}\right), \operatorname{JC}\left(l^{\left(\Psi_{s}^{+}\right)}\right)\right\} \leq 2^{-s / 2}$, where

$$
\begin{aligned}
& \alpha_{\Phi_{s}}^{0}=\liminf _{u \rightarrow 0} \frac{\Phi_{s}^{-1}(u)}{\Phi_{s}^{-1}(2 u)}=\left(\alpha_{\Phi}^{0}\right)^{1-s}\left(\frac{1}{2}\right)^{s / 2}, \\
& \beta_{\Phi_{s}}^{0}=\limsup _{u \rightarrow 0} \frac{\Phi_{s}^{-1}(u)}{\Phi_{s}^{-1}(2 u)}=\left(\beta_{\Phi}^{0}\right)^{1-s}\left(\frac{1}{2}\right)^{s / 2} .
\end{aligned}
$$

EXAMPLE 3.6. Let $\Phi_{r}(u)=e^{|u|^{r}}-1(1<r<\infty)$. For $u \geq 0, \Phi_{r}^{-1}(u)=$ $[\ln (1+u)]^{1 / r}$. If $0<s \leq 1$, then

$$
\Phi_{s}^{-1}(u, r)=[\ln (1+u)]^{(1-s) / r} u^{s / 2} \quad(u \geq 0) .
$$

Note that $C_{\Phi}^{0}=\lim _{t \rightarrow 0} \frac{t \Phi_{r}^{\prime}(t)}{\Phi_{r}(t)}=r$ and $\gamma_{\Phi}^{0}=2^{-1 / C_{\Phi_{r}}^{0}}=2^{-1 / r}$. Hence

$$
\alpha_{\Phi_{s}}^{0}=\beta_{\Phi_{s}}^{0}=\gamma_{\Phi_{s}}^{0}=\lim _{u \rightarrow 0} \frac{\Phi_{s}^{-1}(u, r)}{\Phi_{s}^{-1}(2 u, r)}=\left(\gamma_{\Phi}^{0}\right)^{1-s}\left(\frac{1}{2}\right)^{s / 2}=2^{-(1-s) / r-s / 2} .
$$

By (65) we get

$$
\begin{aligned}
2^{-s / 2} & \geq\left\{\mathrm{JC}\left(l^{\left(\Phi_{s}\right)}\right), \operatorname{JC}\left(l^{\Phi_{s}}\right), \operatorname{JC}\left(l^{\left(\Psi_{s}^{+}\right)}\right), \operatorname{JC}\left(l^{\Psi_{s}^{+}}\right)\right\} \\
& \geq \begin{cases}2^{(1-s) / r+s / 2-1}, & 1<r \leq 2 \\
2^{-(1-s) / r-s / 2}, & 2 \leq r<\infty\end{cases}
\end{aligned}
$$

Since

$$
\lim _{r \searrow 1} \operatorname{JC}\left(l^{\left(\Phi_{s}\right)}\right)=\lim _{r \nearrow \infty} \mathrm{JC}\left(l^{\Phi_{s}}\right)=2^{-s / 2},
$$

for $s=1$ we get $\mathrm{JC}\left(l^{2}\right)=1 / \sqrt{2}$. 
Example 3.7. Let $1<p<\infty$ and $\Phi(u)=|u|^{2 p}+2|u|^{p}$. Then $\Phi \in$ $\Delta_{2}(0) \cap \nabla_{2}(0)$ and $C_{\Phi}^{0}=\lim _{t \rightarrow 0} \frac{t \Phi^{\prime}(t)}{\Phi(t)}=p$. For $u \geq 0$,

$$
\Phi^{-1}(u)=(\sqrt{1+u}-1)^{1 / p} .
$$

For $0<s \leq 1$,

$$
\Phi_{s}^{-1}(u)=(\sqrt{1+u}-1)^{(1-s) / p} u^{s / 2} \quad(u \geq 0) .
$$

Hence

$$
\gamma_{\Phi_{s}}^{0}=\left(\gamma_{\Phi}^{0}\right)^{1-s}\left(\frac{1}{2}\right)^{s / 2}=\left(\frac{1}{2}\right)^{(1-s) / p+s / 2}
$$

By (65) we get

$$
\begin{aligned}
2^{-s / 2} & \geq\left\{\operatorname{JC}\left(l^{\left(\Phi_{s}\right)}\right), \operatorname{JC}\left(l^{\Phi_{s}}\right), \operatorname{JC}\left(l^{\left(\Psi_{s}^{+}\right)}\right), \operatorname{JC}\left(l^{\Psi_{s}^{+}}\right)\right\} \\
& \geq \begin{cases}2^{(1-s) / p+s / 2-1}, & 1<p \leq 2, \\
2^{-(1-s) / p-s / 2}, & 2 \leq p<\infty .\end{cases}
\end{aligned}
$$

Theorem 3.8. Let $\Phi$ be an $\mathcal{N}$-function, $0<s \leq 1$, and let $\Psi_{s}^{+}$be the complementary $\mathcal{N}$-function to $\Phi_{s}$. If $\Phi \notin \Delta_{2}(0) \cap \nabla_{2}(0)$, then

$$
\operatorname{JC}\left(l^{\left(\Phi_{s}\right)}\right)=\operatorname{JC}\left(l^{\Psi_{s}^{+}}\right)=\operatorname{JC}\left(l^{\Phi_{s}}\right)=\operatorname{JC}\left(l^{\left(\Psi_{s}^{+}\right)}\right)=2^{-s / 2} .
$$

Proof. By Corollary 3.5 we only have to prove that for $\Phi \notin \Delta_{2}(0)$ $\cap \nabla_{2}(0)$,

$$
\max \left(\beta_{\Phi_{s}}^{0}, \frac{1}{2 \alpha_{\Phi_{s}}^{0}}\right)=2^{-s / 2}
$$

where

$$
\beta_{\Phi_{s}}^{0}=\left(\beta_{\Phi}^{0}\right)^{1-s}\left(\frac{1}{\sqrt{2}}\right)^{s}, \quad \alpha_{\Phi_{s}}^{0}=\left(\alpha_{\Phi}^{0}\right)^{1-s}\left(\frac{1}{\sqrt{2}}\right)^{s} .
$$

Since $\Phi \notin \Delta_{2}(0) \cap \nabla_{2}(0)$, we have either $\Phi \notin \Delta_{2}(0)$, or $\Phi \notin \nabla_{2}(0)$.

(i) If $\Phi \notin \Delta_{2}(0)$, then $\beta_{\Phi}^{0}=1$ and $1 / 2 \leq \alpha_{\Phi}^{0}$. By (),

$$
\beta_{\Phi_{s}}^{0}=2^{-s / 2}, \quad 2 \alpha_{\Phi_{s}}^{0} \geq 2\left(\frac{1}{2}\right)^{1-s}\left(\frac{1}{\sqrt{2}}\right)^{s}=2^{s / 2} .
$$

Hence

$$
\max \left(\frac{1}{2 \alpha_{\Phi_{s}}^{0}}, \beta_{\Phi_{s}}^{0}\right)=\beta_{\Phi}^{0}=2^{-s / 2} .
$$

(ii) If $\Phi \notin \nabla_{2}(0)$, then $\alpha_{\Phi}^{0}=1 / 2$ and $\beta_{\Phi}^{0} \leq 1$. By (),

$$
\beta_{\Phi_{s}}^{0} \leq 2^{-s / 2}, \quad 2 \alpha_{\Phi_{s}}^{0}=2\left(\frac{1}{2}\right)^{1-s}\left(\frac{1}{\sqrt{2}}\right)^{s}=2^{s / 2} .
$$


Hence

$$
\max \left(\beta_{\Phi_{s}}^{0}, \frac{1}{2 \alpha_{\Phi_{s}}^{0}}\right)=\frac{1}{2 \alpha_{\Phi_{s}}^{0}}=2^{-s / 2} .
$$

Now (67) follows from () and (). Finally, (66) follows from (65) and (67).

EXAMPLE 3.9. Let

$$
M^{-1}(u)= \begin{cases}0, & u=0 \\ (-\ln u)^{-1 / 2} u^{1 / 4}, & 0<u \leq e^{-2} \\ (e / 2)^{1 / 2} u^{1 / 2}, & e^{-2} \leq u<\infty\end{cases}
$$

Then

$$
\mathrm{JC}\left(l^{(M)}\right)=\operatorname{JC}\left(l^{M}\right)=\operatorname{JC}\left(l^{(N)}\right)=\operatorname{JC}\left(l^{N}\right)=\frac{1}{\sqrt[4]{2}} .
$$

In fact (see Kamińska [8, p. 304]), define the $\mathcal{N}$-function

$$
\Phi(u)= \begin{cases}0, & u=0, \\ e^{-1 /|u|}, & |u| \in(0,1 / 2], \\ 4 e^{-2} u^{2}, & |u| \in[1 / 2, \infty) .\end{cases}
$$

Since

$$
C_{\Phi}^{0}=\lim _{t \rightarrow 0} \frac{t \phi(t)}{\Phi(t)}=\lim _{t \rightarrow 0} \frac{1}{t}=\infty,
$$

we have $\Phi \notin \Delta_{2}(0)$. For $u \geq 0$,

$$
\Phi^{-1}(u)= \begin{cases}0, & u=0 \\ (-\ln u)^{-1}, & 0<u \leq e^{-2} \\ (e / 2) u^{1 / 2}, & e^{-2} \leq u<\infty\end{cases}
$$

We get $M^{-1}(u)=\left[\Phi_{s}^{-1}(u)\right]_{s=1 / 2}$. By Theorem 3.8 we get ().

Example 3.10. Rao and Ren [13] define an $\mathcal{N}$-function $\Phi$ to be the inverse of

$$
\Phi^{-1}(u)= \begin{cases}0, & u=0 \\ u \ln (1 / u), & 0<u \leq e^{-2} \\ (2 / e) u^{1 / 2}, & e^{-2} \leq u<\infty .\end{cases}
$$

Since $\gamma_{\Phi}^{0}=\lim _{u \rightarrow 0} \frac{\Phi^{-1}(u)}{\Phi^{-1}(2 u)}=\frac{1}{2}$, by Theorem 1.5 we have $\Phi \notin \nabla_{2}(0)$. For $0<s \leq 1$,

$$
\Phi_{s}^{-1}(u)=\left[\Phi^{-1}(u)\right]^{1-s}\left[\frac{1}{\sqrt{2 u}}\right]^{s}= \begin{cases}0, & u=0, \\ (u \ln u)^{1-s} u^{s / 2}, & 0<u \leq e^{-2}, \\ (2 / e)^{1-s} u^{1 / 2}, & e^{-2} \leq u<\infty .\end{cases}
$$

If $\Psi_{s}^{+}$is the complementary $\mathcal{N}$-function to $\Phi_{s}$, then by Theorem 3.8 we get

$$
\mathrm{JC}\left(l^{\left(\Phi_{s}\right)}\right)=\mathrm{JC}\left(l^{\Psi_{s}^{+}}\right)=\mathrm{JC}\left(l^{\Phi_{s}}\right)=\mathrm{JC}\left(l^{\left(\Psi_{s}^{+}\right)}\right)=2^{-s / 2} .
$$




\section{References}

[1] D. Amir, On Jung's constant and related constants in normed linear spaces, Pacific J. Math. 118 (1985), 1-15.

[2] W. L. Bynum, Normal structure coefficients for Banach space, Pacific J. Math. 86 (1980), 427-435.

[3] S. T. Chen, Geometry of Orlicz spaces, Dissertationes Math., 356 (1996).

[4] C. E. Cleaver, On the extension of Lipschitz-Hölder maps on Orlicz spaces, Studia Math. 42 (1972), 195-204.

[5] S. W. Golomb and L. D. Baumert, The search for Hadamard matrices, Amer. Math. Monthly 70 (1963), 12-17.

[6] V. I. Ivanov and S. A. Pichugov, Jung constants of the $l_{p}^{n}$-spaces, Math. Notes 48 (1990), 997-1004.

[7] H. W. E. Jung, Über die kleinste Kugel, die eine raümliche Figur einschliesst, J. Reine Angew. Math. 123 (1901), 241-257.

[8] A. Kamińska, Strict convexity of sequence Musielak-Orlicz spaces with Orlicz norm, J. Funct. Anal. 50 (1983), 285-305.

[9] M. A. Krasnosel'skiı̌ and Ya. B. Rutickiŭ, Convex Functions and Orlicz Spaces, Noordhoff, Groningen, 1961.

[10] J. Lindenstrauss and L. Tzafriri, Classical Banach Spaces, I and II, Springer, Berlin, 1977 and 1979.

[11] E. Maluta, Uniformly normal structure and related coefficients, Pacific J. Math. 111 (1984), 357-369.

[12] S. A. Pichugov, Jung's constant for the space $L^{p}$, Math. Notes 43 (1988), 348-353.

[13] M. M. Rao and Z. D. Ren, Theory of Orlicz Spaces, Dekker, New York, 1991.

Department of Mathematics

TongJi University

Shanghai 200092, China

E-mail: abc7212@163.com 